\title{
Highly Eribulin-resistant KBV20C Oral Cancer Cells Can Be Sensitized by Co-treatment with the Third-generation P-Glycoprotein Inhibitor, Elacridar, at a Low Dose
}

\author{
YUJIN PARK*, JI-YEON SON*, BYUNG-MU LEE, HYUNG SIK KIM and SUNGPIL YOON \\ School of Pharmacy, Sungkyunkwan University, Suwon, Republic of Korea
}

\begin{abstract}
Background/Aim: Eribulin mesylate, also called Halaven $^{\circledR}$ (HAL), was recently developed as a microtubuletargeting drug and is used in the clinic for resistant or metastatic cancer. Previously, we showed that P-glycoprotein $(P$-gp)-overexpressing KBV20C oral cancer cells are highly resistant to $H A L$ compared to sensitive $K B$ cells. This qualitative study was designed to identify specific $P-g p$ inhibitors that increase the sensitivity of highly resistant cancer cells to HAL. Materials and Methods: In order to identify functional P-gp inhibitors, HAL-treated KBV20C cells were co-treated with P-gp inhibitors, verapamil, elacridar, cyclosporine A, mitotane, piperine, fumagillin, curcumin, indomethacin, probenecid, sulindac, tesmilifene, and $C-4$. We then evaluated which P-gp inhibitors required a low dose to sensitize KBV20C cells to HAL. We also determined whether a low dose of a P-gp inhibitor could inhibit P-gp efflux pumping. Results: We found that cyclosporine A sensitized HAL-treated KBV20C cells at a low dose, whereas verapamil, another first-generation P-gp inhibitor, required a dose that was nearly 10-fold higher. We also found that the natural products, piperine and mitotane, sensitized KBV2OC cells to HAL co-treatment. Interestingly, we found that elacridar, a third-generation P-gp inhibitor, sensitized HAL-treated cells at a low dose. Elacridar required approximately a 500-fold lower dose than that of verapamil to exert a similar effect. All inhibitors showed $P$-gp inhibitory activity that correlated with sensitivity to HAL. Conclusion: These results suggest that highly HAL-
\end{abstract}

*These Authors contributed equally to this study.

Correspondence to: Sungpil Yoon, Ph.D., School of Pharmacy, Sungkyunkwan University, 2066 Seobu-ro, Jangan-gu, Suwon, Gyeonggi-do, 16419, Republic of Korea. Tel: +82 312907789, Fax: +82 312928800, e-mail: syoon88@gmail.com

Key Words: Halaven, verapamil, elacridar, cyclosporine A, P-gp inhibitors, drug-resistance. resistant cancer cells can be sensitized with cyclosporine A or elacridar, specific P-gp inhibitors that exert their effects at a low dose. These findings provide important information regarding the sensitization of highly HAL-resistant cells with selective P-gp inhibitors and indicate that elacridar may be used to treat such highly HAL-resistant cancer cells.

Anti-mitotic drugs, which target different binding sites on tubulin, are widely used for treating different types of cancer $(1,2)$. These compounds inhibit mitosis by targeting microtubules and preventing their polymerization or depolymerization $(3,4)$. Eribulin, also called Halaven ${ }^{\circledR}$ (HAL), was recently developed and used in the clinic to treat resistant or metastatic cancer. HAL has been developed to overcome the resistance of cancer cells to routinely used anti-mitotic drugs. It targets the depolymerization of microtubules $(5,6)$. HAL is considered a promising drug for triple-negative breast cancer and certain resistant cancer types (7-9). Since patients are expected to develop resistance to HAL, identifying the mechanism(s) that underlie cell sensitization would be an important step in the development of more effective treatments by designing approaches to increase HAL-associated apoptosis.

P-Glycoprotein (P-gp) overexpression is a well-known mechanism for anticancer drug resistance (10, 11). P-gp plays a role in such resistance by actively pumping anticancer drugs out of the cell. Although P-gp inhibitors have been developed, toxicity in normal cells resulted in their failure in clinical testing. After the toxicity of firstgeneration P-gp inhibitors was established, several trials using other drugs were conducted $(12,13)$. Natural products or pre-existing clinical drugs with $\mathrm{P}$-gp inhibitory activity were tested $(4,13-15)$, since they had already passed drugtoxicity screening. Other strategies involved modifying the side-chains of known P-gp inhibitors. Non-competitive inhibitors were also designed with computational simulations and are called third-generation P-gp inhibitors (12, 13). All of these were designed to minimize toxicity in normal cells. Since highly HAL-resistant cancer cells use P-gp efflux 
mechanisms, it is important to test which $\mathrm{P}$-gp inhibitors adequately and specifically inhibit the ability of P-gp to pump out HAL.

Therefore, we performed this study to increase our understanding of the mechanisms associated with sensitization of HAL-resistant KBV20C oral cancer cells overexpressing P-gp (16). Mainly, we focused on identifying specific P-gp inhibitors for sensitizing highly HAL-resistant KBV20C cells at low doses. We selected different known Pgp inhibitors $(4,10-13,15,17,18)$ which have been used or suggested in clinical trials. We then tested which P-gp inhibitors could sensitize HAL-treated KBV20C cells at low dose. We also tested whether these agents could inhibit the ability of P-gp to act as an efflux pump in KBV20C cells. Since these drugs have been considered for use in the treatment of humans, they could be readily available for clinical use once their HAL synergistic activities are better understood. These results will contribute to the development of P-gp inhibitor-based therapies for the co-treatment of highly HAL-resistant tumors.

\section{Materials and Methods}

Reagents. Elacridar, piperine, mitotane, indomethacin, cyclosporine A, and probenecid was purchased from Santa Cruz Biotechnology (Dallas, TX, USA). Fumagillin and sulindac were purchased from Enzo Life Sciences (Farmingdale, NY, USA). Verapamil and tesmilifene were purchased from Sigma-Aldrich (St. Louis, MO, USA). Curcumin and C-4 were supplied by Calbiochem (Bellerica, MA, USA). Calcein-AM was obtained from Invitrogen (Carlsbad, CA, USA). Aqueous solutions of HAL (Eisai Korea, Seoul, Republic of Korea) were obtained from the National Cancer Center in South Korea.

Cell culture. Human oral squamous cell carcinoma cell line KB and its multidrug-resistant subline, KBV20C, were obtained from Dr. Yong Kee Kim (College of Pharmacy, Sookmyung Women's University, Seoul, Republic of Korea) and have been previously described (17, 19, 20). All cell lines were cultured in RPMI-1640 containing $10 \%$ fetal bovine serum, $100 \mathrm{U} / \mathrm{ml}$ penicillin and $100 \mu \mathrm{g} / \mathrm{ml}$ streptomycin (WelGENE, Daegu, South Korea).

Microscopic observation. Cells grown in 6-well plates were treated with the drugs under study, the medium was removed and phosphate-buffered saline (PBS) was added to each plate. Cells were examined immediately in two independent experiments using an Axio observer.Z1 fluorescence inverted microscope (Carl Zeiss, Oberkochen, Germany) with a $5 \times$ or $10 \times$ objective lens (Carl Zeiss EC Plan-Neofluar).

Calcein-AM uptake tests. Calcein-AM uptake was used to determine the ability of agents to inhibit P-gp using a previously described method (21-26). Briefly, cells grown in 6-well plates were treated with study drugs and incubated for $24 \mathrm{~h}$ at $37^{\circ} \mathrm{C}$. Cells were then incubated with $0.1 \mu \mathrm{g} / \mathrm{ml}$ Calcein-AM for $1 \mathrm{~h} 30 \mathrm{~min}$ at $37^{\circ} \mathrm{C}$. The medium was then removed, and the cells were washed with PBS. Stained cells were analyzed using a FACSCalibur flow cytometric system (BD Biosciences, Franklin Lakes, NJ, USA). In this experiment, cellular accumulation of green fluorescence was indicative of intracellular accumulation of calcein-AM and an effect on P-gp inhibition. We performed two independent experiments.

\section{Results}

Co-treatment with low-dose cyclosporine A sensitizes highly $H A L$-resistant KBV20C cells. KBV20C cells present an antimitotic drug-resistant phenotype via P-gp overexpression (21, 22). We previously showed that the concentration of HAL required for a similar response was approximately 500-fold higher than that required for the parental drug-sensitive KB cells (16). Considering that our previous studies showed less than 10-fold differences when other anti-mitotic drugs were used (20, 23-26), KBV20C cell line is a very useful model of highly HAL-resistant cancer cells. In this study, we tested whether P-gp-overexpressing HAL-resistant cancer cells can be selectively sensitized by co-treatment with known P-gp inhibitors.

Firstly, we tested the well-known, first-generation P-gp inhibitor, verapamil $(12,13)$, in HAL-treated KBV20C cells. As shown in Figure 1A, co-treatment with $50 \mu \mathrm{M}$ HAL and $5 \mu \mathrm{M}$ verapamil increased sensitization of drug-resistant KBV20C cells, confirming that HAL-resistance in KBV20C cells involves efflux pumping associated with $\mathrm{P}$-gp overexpression. We then tested another known firstgeneration P-gp inhibitor, cyclosporine A (11). We found that 10-fold lower concentration of cyclosporine A sensitized the cells to a similar degree as verapamil did (Figure 1B). The results suggest that cyclosporine $\mathrm{A}$ is more effective in sensitizing highly HAL-resistant cancer cells than verapamil. We also tested whether both verapamil and cyclosporine A could exert their effects using reduced concentrations of HAL. As seen in Figure 1C-D, $10 \mu \mathrm{M}$ or $5 \mu \mathrm{M}$ of HAL had similar effects to those observed with $10 \mu \mathrm{M}$ or $20 \mu \mathrm{M}$ verapamil and $1 \mu \mathrm{M}$ or $2 \mu \mathrm{M}$ cyclosporine $\mathrm{A}$. This suggests that the HAL dose for co-treatment can also be reduced when low-dose cyclosporine $\mathrm{A}$ is used against highly HALresistant cancer cells.

We also evaluated P-gp-inhibitory activity. As shown in Figure 1E, cyclosporine A has a higher P-gp-inhibitory activity than verapamil does, suggesting that cyclosporine $\mathrm{A}$ has greater specificity for P-gp inhibition of HAL efflux. Altogether, we found that cyclosporine A can be used at a low dose, since it has selectivity for highly HAL-resistant cancer cells and increased inhibition of P-gp.

A high dose of piperine and mitotane sensitizes HAL-treated KBV20C cells. In order to reduce toxicity of P-gp inhibitors, natural products have been tested $(14,15,27)$. Among them, we tested whether curcumin, fumagillin, and piperine could sensitize HAL-treated KBV20C cells. As shown in Figure 

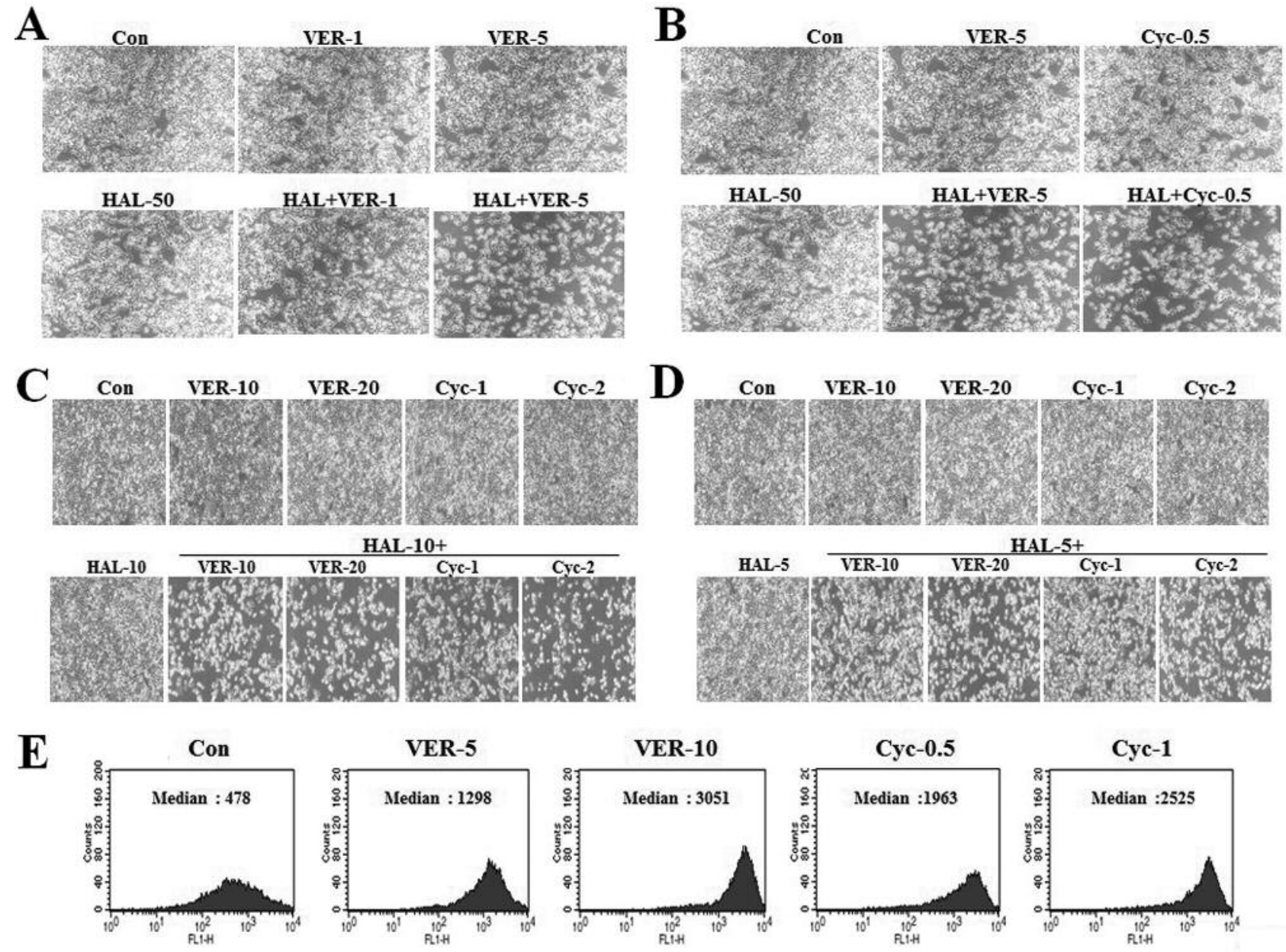

Figure 1. At low dose, cyclosporine A, a first-generation P-glycoprotein (P-gp) inhibitor, sensitizes highly Halaven (HAL)-resistant KBV20C cells. A-D: KBV20C cells were grown on 6-well plates and treated with different micromolar concentrations (indicated as suffix) of verapamil (VER), cyclosporine A (Cyc) alone and in combination with $50 \mathrm{ng} / \mathrm{ml} \mathrm{HAL} \mathrm{(10} \mathrm{ng} / \mathrm{ml} \mathrm{or} 5 \mathrm{ng} / \mathrm{ml} \mathrm{HAL})$ or with $0.1 \%$ dimethylsulfoxide (DMSO) (Con). After 1 day, all cells were observed using an inverted microscope at $\times 50$ magnification. E: KBV20C cells were grown on 6-well plates and treated with $5 \mu \mathrm{M}$ or $10 \mu \mathrm{M}$ verapamil, $0.5 \mu \mathrm{M}$ or $1 \mu \mathrm{M}$ cyclosporine A, or $0.1 \%$ DMSO (Con). After $24 \mathrm{~h}$, all cells were stained with calcein-AM, as described in the Materials and Methods. The stained cells were subsequently examined by using FACS analysis.

$2 \mathrm{~A}$ and $\mathrm{B}$, we found that $100 \mu \mathrm{M}$ piperine increased sensitivity to HAL, whereas neither curcumin nor fumagillin had this effect. In the case of curcumin, a single treatment with $50 \mu \mathrm{M}$ sensitized KBV20C cells to HAL (Figure 2C), but co-treatment with HAL produced no additional effect. This suggests that curcumin does not inhibit $\mathrm{P}$-gp activity in highly HAL-resistant cancer cells.

Although a relatively high dose of piperine was needed to overcome HAL-resistance, considering that natural products are relatively harmless to normal tissues, it could be possible to use piperine in combination with HAL in patients.

In order to reduce toxicity, third-generation P-gp inhibitors have been developed $(12,13)$. Previous reports have suggested that these inhibitors can target P-gp at very low doses $(12,13)$. Therefore, we also tested these inhibitors (namely, mitotane, probenecid, sulindac, and tesmilifene) in highly HAL-resistant KBV20C cells. As shown in Figure 2B-D, we found that only mitotane was able to sensitize HAL-treated KBV20C cells. In previous reports, indomethacin, probenecid, and C-4 showed P-gp inhibitory activities $(12,13,17)$; therefore, we tested whether they could also increase sensitivity to HAL co-treatment. However, as shown in Figure 2D-F, they did not increase sensitivity. Taken together, we showed that the natural product, piperine and the clinical drug, mitotane, sensitize highly HAL-resistant cancer cells. 


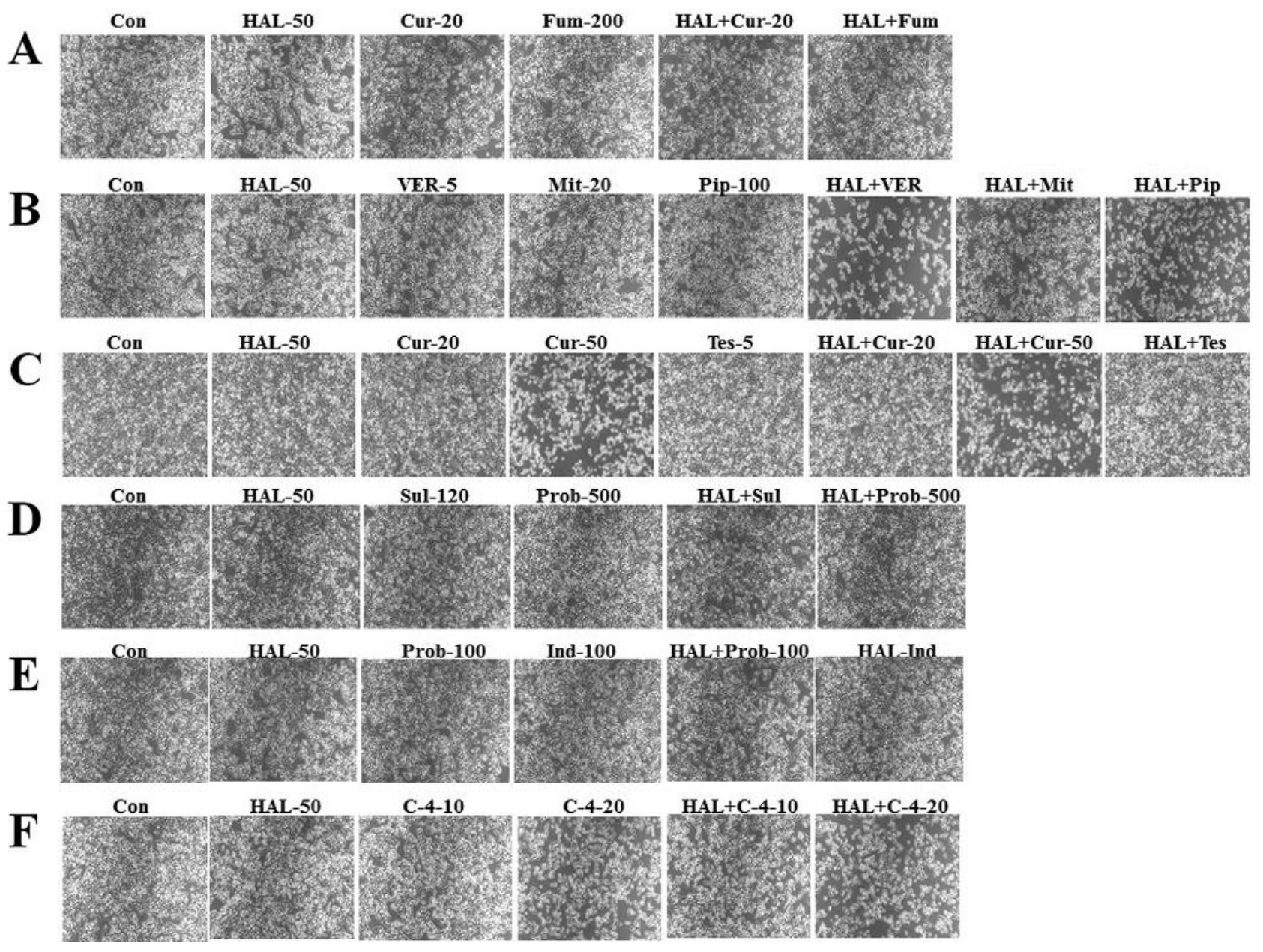

Figure 2. Natural product P-glycoprotein (P-gp) inhibitors piperine and mitotane sensitize highly Halaven (HAL)-treated KBV20C cells. A-F: KBV20C cells were grown on 6-well plates and treated with different micromolar concentrations (indicated as suffix) of curcumin (Cur), fumagillin (Fum), verapamil (VER), mitotane (Mit), piperine (Pip), tesmilifene (Tes), sulindac (Sul), probenecid (Prob), indomethacin (Ind), or C-4 alone and in combination with 50 $\mathrm{ng} / \mathrm{ml}$ HAL (HAL), or with $0.1 \%$ DMSO (Con). After 1 day, all cells were observed using an inverted microscope at $\times 50$ magnification.

Ability of P-gp inhibition correlates with degree of sensitization HAL-resistant KBV20C cells. We compared the sensitizing effects for verapamil, mitotane, and piperine in HAL-treated KBV20C cells (Figure 3A). We found that mitotane needed a 5 -fold higher dose than verapamil did to achieve a similar effect. In the case of piperine, for a similar sensitizing effect, an almost 10-fold higher dose than that of verapamil was needed (Figure 3A). Both mitotane and piperine inhibited P-gp activity (Figure 3B), but their inhibitory activity was much lower than that of verapamil or cyclosporine A. We also found that other drugs (mitotane, piperine, fumagillin, curcumin, indomethacin, probenecid, sulindac, tesmilifene, and C-4) exhibited little P-gpinhibitory activity, suggesting that HAL-resistant KBV20C cells can be sensitized only by co-treatment with the P-gp inhibitors verapamil, mitotane, and piperine.
Low-dose elacridar has a highly selective sensitizing effect on HAL-resistant cancer cells. During our screening of potential P-gp inhibitors, we found a very specific thirdgeneration P-gp inhibitor elacridar was able to sensitize highly HAL-resistant KBV20C cancer cells (Figure 4A). The effects of HAL co-treatment $(10 \mu \mathrm{M})$ were sensitized with $0.01 \mu \mathrm{M}$ elacridar, suggesting that toxicity of the drug combination could be minimized. We also found that HAL concentrations could be further reduced, down to $1 \mu \mathrm{M}$, when $0.05 \mu \mathrm{M}$ elacridar was used (Figure 4B). When we compared it to cyclosporine A, more than a 50-fold lower dose of elacridar was able to sensitize KBV20C cells to HAL treatment, suggesting that toxicity could be minimized when co-treating resistant cancer cells with HAL and elacridar.

We next tested the P-gp-inhibiting ability of elacridar. We found that elacridar showed high P-gp-inhibiting ability 
A
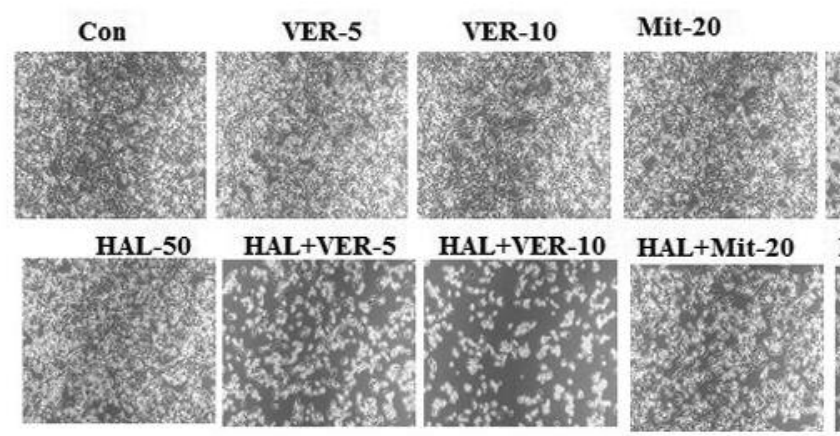

Mito-50

Pip-50

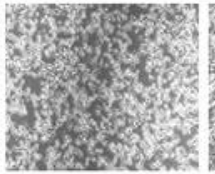

HAL+Mit-50

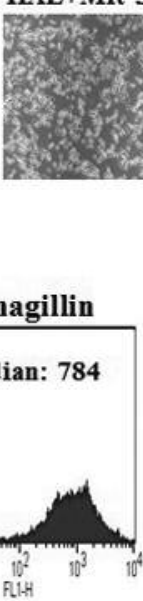

Probenecid
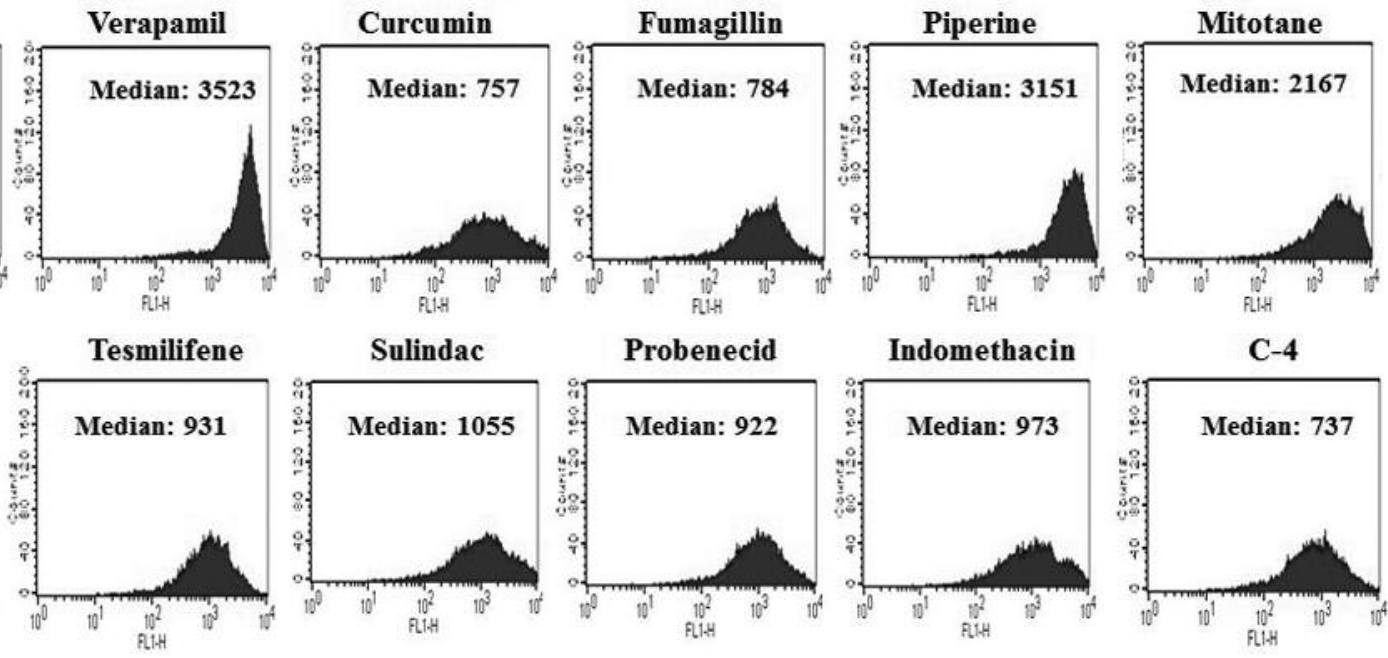

Figure 3. Inhibitory ability of known P-glycoprotein (P-gp) inhibitors correlates with sensitizing Halaven (HAL)-treated KBV20C cells. A: KBV20C cells were grown on 6-well plates and treated with different micromolar concentrations (indicated as suffix) of verapamil (VER), mitotane (Mit), or piperine (Pip) alone and in combination with $50 \mathrm{ng} / \mathrm{ml} \mathrm{HAL}$, or with $0.1 \%$ dimethylsulfoxide (DMSO) (Con). After 1 day, all cells were observed using an inverted microscope at $\times 50$ magnification. B: KBV20C cells were grown on 6-well plates and treated with $10 \mu M$ verapamil, $20 \mu M$ curcumin, $200 \mu \mathrm{M}$ fumagillin, $100 \mu \mathrm{M}$ piperine, $50 \mu \mathrm{M}$ mitotane, $5 \mu \mathrm{M}$ tesmilifene, $60 \mu \mathrm{M}$ sulindac, $100 \mu \mathrm{M}$ probenecid, $100 \mu \mathrm{M}$ indomethacin, $10 \mu M$ C-4, or 0.1\% DMSO (Con). After 24 h, all cells were stained with calcein-AM, as described in the Materials and Methods section. The stained cells were subsequently examined by FACS analysis.

(Figure 4D). However, when comparing the sensitizing effects between elacridar and cyclosporine $\mathrm{A}$, we found that the sensitizing dose of elacridar led to less P-gp inhibition than the sensitizing dose of cyclosporine A did (Figure 4D). This suggests that the effect of elacridar on highly HAL-resistant cancer cells involves both P-gp-inhibit ion and other unknown mechanisms. Overall, we identified elacridar as being most suitable for sensitizing highly resistant cancer cells to HAL.

\section{Discussion}

HAL was recently developed and is especially promising for the treatment of patients for whom anticancer drugs had previously failed $(5,7-9)$. Since it is expected that patients with cancer treated with HAL will ultimately develop resistance to HAL, we investigated sensitizing mechanisms and drugs for HAL-resistant cancer. Previously, we identified that P-gp-overexpressing KBV20C cells are highly HALresistant (16). Therefore, KBV20C cells are very useful as models of highly HAL-resistant cancer. We assumed that our studies using KBV20C cancer cells could provide insight for the treatment of patients with HAL-resistant cancer.

Since the efflux of HAL by P-gp is the main mechanism for the resistance of KBV20C cells to HAL, we tried to identify effective P-gp inhibitors to co-treat with HAL. Since P-gp inhibitors have shown toxicity in normal cells, we tried to 

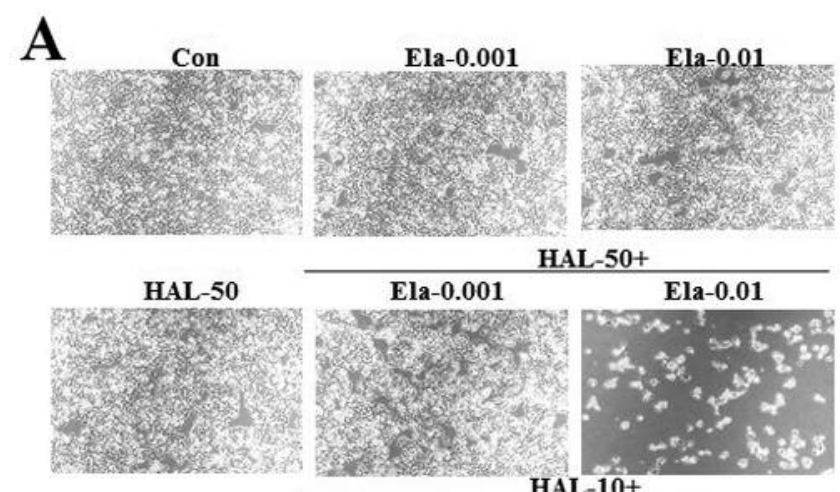

HAL-10
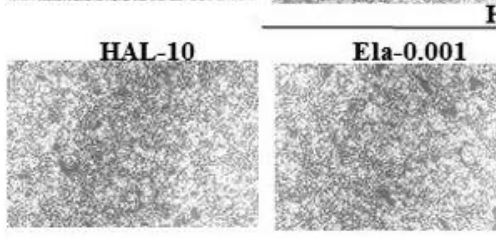

HAL-10+

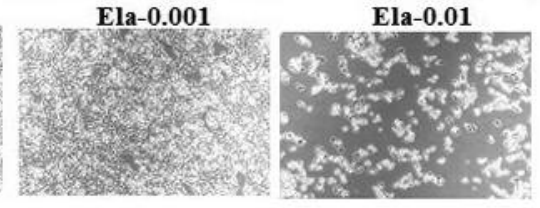

B
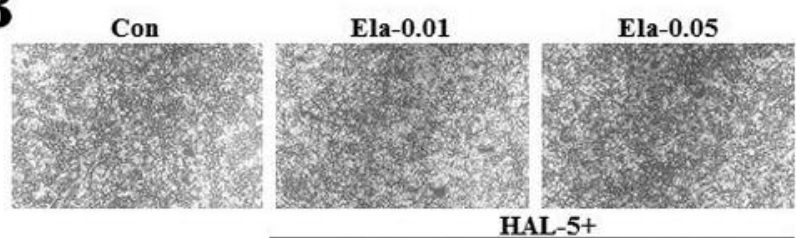

HAL-5
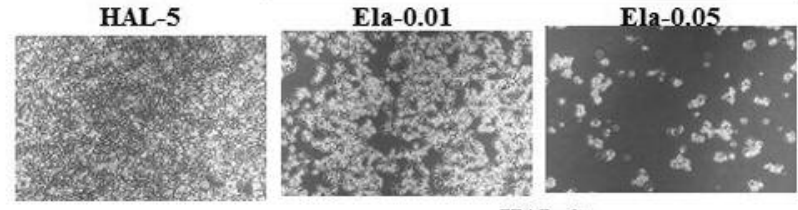

HAL-1+
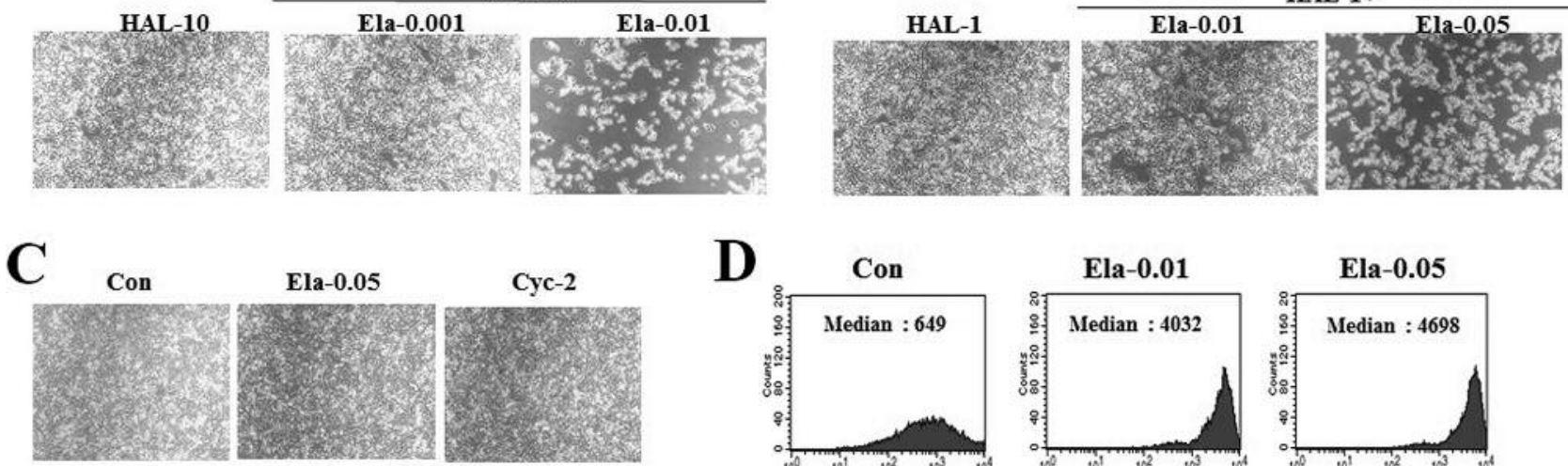

Ela-0.05

Cyc-2

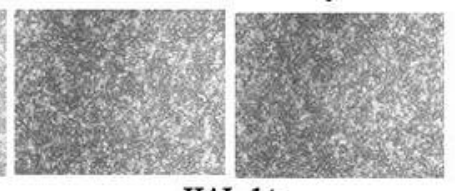

HAL-1+

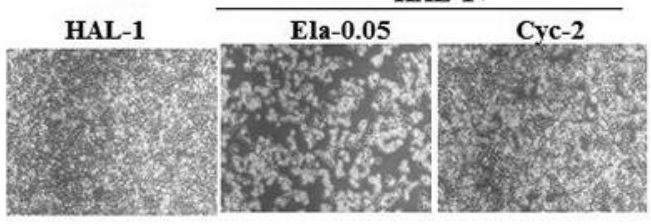

D
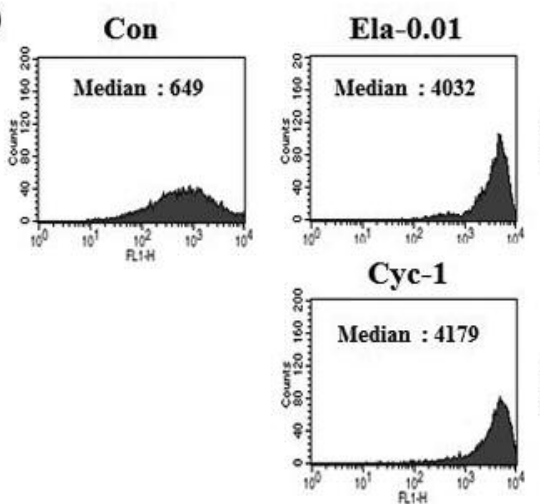

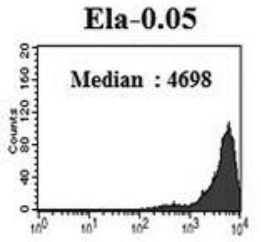

Cyc-2

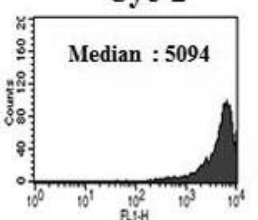

Figure 4. Low-dose elacridar has a highly selective sensitization-effect on Halaven (HAL)-resistant cancer cells. A-C: KBV20C cells were grown on 6-well plates and treated with different micromolar concentrations (indicated as suffix) of elacridar (Ela), cyclosporine A (Cyc) alone and in combination with $50 \mathrm{ng} / \mathrm{ml} \mathrm{HAL} \mathrm{(10} \mathrm{ng} / \mathrm{ml}, 5 \mathrm{ng} / \mathrm{ml}$, or $1 \mathrm{ng} / \mathrm{ml} \mathrm{HAL})$ or with $0.1 \%$ dimethylsulfoxide (DMSO) (Con). After 1 day, all cells were observed using an inverted microscope at $\times 50$ magnification. D: KBV20C cells were grown on 6-well plates and treated with $0.01 \mu M$ or $0.05 \mu M$ elacridar, $1 \mu \mathrm{M}$ or $2 \mu \mathrm{M}$ cyclosporine A, or $0.1 \%$ DMSO (Con). After 24 h, all cells were stained with calcein-AM, as described in the Materials and Methods section. The stained cells were subsequently examined by FACS analysis.

identify low-dose P-gp inhibitors for sensitizing when combined with HAL. In order to identify selective P-gp inhibitors, we chose those used in clinical trials $(12,13)$ and tested them in co-treatment with HAL in KBV20C cells. We assumed that drugs already in clinical trials could be easily given to patients with HAL-resistant cancer. We selected and tested the P-gp inhibitors, verapamil, cyclosporine A, fumagillin, curcumin, piperine, tesmilifene, mitotane, sulindac, and elacridar (11-15, 18). They can be categorized as first-generation, thirdgeneration, and natural products, in order of decreasing toxicity $(12,13)$. In addition, we tested known P-gp inhibitors (namely indomethacin, probenecid, and C-4) from prior literature reports $(11-13,17)$, but we did not obtain positive results with these.
Initially, we tested the first-generation P-gp inhibitors, verapamil and cyclosporine A. Cyclosporine A required a $\sim 10$-fold lower dose than verapamil did for similar sensitization of HAL-treated KBV20C cells. Although firstgeneration P-gp inhibitors had previously shown high toxicity at high dose $(12,13)$, we assume that cyclosporine A can be applied at lower dose in HAL-resistant cancer cells.

Secondly, when natural products (namely fumagillin, curcumin, and piperine) were tested, we found that only piperine increased sensitivity of HAL-resistant cancer cells to HAL. However, a more than 10-fold higher dose of piperine was required for an effect similarto that of verapamil. Considering that natural products are relatively 
benign against normal tissue and minimize toxicity, piperine can be used for overcoming HAL resistance.

Lastly, we tested third-generation P-gp inhibitors (namely tesmilifene, mitotane, sulindac, elacridar). We did not find sensitization of HAL-treated KBV20C cells with tesmilifene and sulindac, whereas mitotane and elacridar showed P-gp inhibition of HAL-resistant KBV20C cells. Considering that third-generation $\mathrm{P}$-gp inhibitors are designed to minimize toxicity with low dose, it was surprising that mitotane required a high dose; more than a 5-fold higher dose of mitotane was required for sensitization similar to that of verapamil. Most importantly, we identified elacridar, which needs a very low dose to inhibit P-gp in HAL-resistant KBV20C cells. A dose as low as $0.01 \mu \mathrm{M}$ elacridar was enough to increase sensitivity of HAL-resistant KBV20C cells. When compared to that of verapamil, elacridar was needed at only a $<500$-fold lower dose. These results suggest that elacridar is highly specific for inhibition of P-gp in HAL-resistant cancer cells. We also found that HAL concentrations can be reduced with co-treatment of elacridar, suggesting that HAL toxicity can be minimized.

When we tested P-gp inhibition with recognized P-gp inhibitors (namely verapamil, cyclosporine A, piperine, mitotane, and elacridar) using HAL-resistance, we found that P-gp inhibition corresponded to the sensitizing concentrations of the drugs. This suggests that P-gp inhibition plays a major role in sensitizing KBV20C cells to co-treatment with HAL.

However, elacridar seems to have activity through other mechanisms beyond P-gp inhibition, since P-gp inhibition cannot fully explain the highly selective sensitizing effect of elacridar on KBV20C cells. Therefore, more mechanistic studies of elacridar are needed. In our current microscopic analysis, we focused on qualitative data to reveal effects of P-gp inhibitors. In future studies, we need to demonstrate sensitization-mechanisms by analyzing quantitative data such as from MTS, FACS, annexin V, and western-blot analysis.

Collectively, we identified five P-gp inhibitors for sensitizing highly HAL-resistant cancer cells, namely verapamil, cyclosporine A, piperine, mitotane, and elacridar. We assume that these drugs might be used to overcome cancer cell resistance to HAL. Most importantly, we found that elacridar has high selectivity for sensitizing HAL-resistant cancer cells. Since these drugs are already used in clinical settings, the urgent need for pharmacological treatments of HAL-resistant cancer can be efficiently addressed, and these drugs may be used to treat patients with HAL-resistant tumors at a faster pace.

\section{Conflicts of Interests}

The Authors declare no conflict of interests in regard to this study.

\section{Acknowledgements}

The Authors thank Ae-Ran Choi and Ju-Hwa Kim for help in technical support and preparation of this article. This research was supported by the National Research Foundation of Korea (NRF) funded by the Korean government (NRF-2016R1A4A1011189 \& NRF-2017R1D1A1B03029158).

\section{References}

1 Jordan MA and Wilson L: Microtubules as a target for anticancer drugs. Nat Rev Cancer 4: 253-265, 2004.

2 McGrogan BT, Gilmartin B, Carney DN and McCann A: Taxanes, microtubules and chemoresistant breast cancer. Biochim Biophys Acta 1785: 96-132, 2008.

3 Matsuo K, Bond VK, Im DD and Rosenshein NB: Prediction of chemotherapy response with platinum and taxane in the advanced stage of ovarian and uterine carcinosarcoma: a clinical implication of in vitro drug resistance assay. Am J Clin Oncol 33: 358-363, 2010

4 Yang H and Dou QP: Targeting apoptosis pathway with natural terpenoids: implications for treatment of breast and prostate cancer. Curr Drug Targets 11: 733-744, 2010.

5 Dell'Ova M, De Maio E, Guiu S, Roca L, Dalenc F, Durigova A, Pinguet F, Bekhtari K, Jacot W and Pouderoux S: Tumour biology, metastatic sites and taxanes sensitivity as determinants of eribulin mesylate efficacy in breast cancer: results from the ERIBEX retrospective, international, multicenter study. BMC Cancer 15: $659,2015$.

6 Laughney AM, Kim E, Sprachman MM, Miller MA, Kohler RH, Yang KS, Orth JD, Mitchison TJ and Weissleder R: Single-cell pharmacokinetic imaging reveals a therapeutic strategy to overcome drug resistance to the microtubule inhibitor eribulin. Sci Transl Med 6: 261ra152, 2014

7 Aogi K, Iwata H, Masuda N, Mukai H, Yoshida M, Rai Y, Taguchi K, Sasaki Y and Takashima S: A phase II study of eribulin in Japanese patients with heavily pretreated metastatic breast cancer. Ann Oncol 23: 1441-1448, 2012.

8 Dybdal-Hargreaves NF, Risinger AL and Mooberry SL: Eribulin mesylate: mechanism of action of a unique microtubule-targeting agent. Clin Cancer Res 21: 2445-2452, 2015.

9 Inoue K, Saito T, Okubo K, Kimizuka K, Yamada H, Sakurai T, Ishizuna K, Hata S, Kai T and Kurosumi M: Phase II clinical study of eribulin monotherapy in Japanese patients with metastatic breast cancer who had well-defined taxane resistance. Breast Cancer Res Treat 157: 295-305, 2016.

10 Chen Z, Shi T, Zhang L, Zhu P, Deng M, Huang C, Hu T, Jiang $\mathrm{L}$ and Li J: Mammalian drug efflux transporters of the ATP binding cassette $(\mathrm{ABC})$ family in multidrug resistance: A review of the past decade. Cancer Lett 370: 153-164, 2016.

11 Chufan EE, Kapoor K and Ambudkar SV: Drug-protein hydrogen bonds govern the inhibition of the ATP hydrolysis of the multidrug transporter P-glycoprotein. Biochem Pharmacol 101: 40-53, 2016.

12 Shukla S, Wu CP and Ambudkar SV: Development of inhibitors of ATP-binding cassette drug transporters: present status and challenges. Expert Opin Drug Metab Toxicol 4: 205223, 2008.

13 Yang K, Wu J and Li X: Recent advances in the research of Pglycoprotein inhibitors. Biosci Trends 2: 137-146, 2008. 
14 Guseman AJ, Miller K, Kunkle G, Dively GP, Pettis JS, Evans JD, vanEngelsdorp D and Hawthorne DJ: Multi-drug resistance transporters and a mechanism-based strategy for assessing risks of pesticide combinations to honey bees. PLoS One 11: e0148242, 2016.

15 Neerati P, Sudhakar YA and Kanwar JR: Curcumin regulates colon cancer by inhibiting P-glycoprotein in in situ cancerous colon perfusion rat model. J Cancer Sci Ther 5: 313-319, 2013.

16 Cheon JH, Lee BM, Kim HS and Yoon S: Highly Halavenresistant KBV20C cancer cells can be sensitized by co-treatment with fluphenazine. Anticancer Res 36: 5867-5874, 2016.

17 Kim YK, Song YJ, Seo DW, Kang DW, Lee HY, Rhee DK, Han JW, Ahn CM, Lee S and Kim SN: Reversal of multidrug resistance by 4-chloro- $N$-(3-((E)-3-(4-hydroxy-3-methoxyphenyl)acryloyl) phenyl)benzamide through the reversible inhibition of $\mathrm{P}$ glycoprotein. Biochem Biophys Res Commun 355: 136-142, 2007.

18 Xia CQ and Smith PG: Drug efflux transporters and multidrug resistance in acute leukemia: therapeutic impact and novel approaches to mediation. Mol Pharmacol 82: 1008-1021, 2012.

19 Kim H, Kim SN, Park YS, Kim NH, Han JW, Lee HY and Kim YK: HDAC inhibitors down-regulate $M R P 2$ expression in multidrug resistant cancer cells: implication for chemosensitization. Int J Oncol 38: 807-812, 2011.

$20 \mathrm{Kim}$ JH, Chae M, Choi AR, Sik Kim H and Yoon S: SP600125 overcomes antimitotic drug-resistance in cancer cells by increasing apoptosis with independence of P-gp inhibition. Eur J Pharmacol 723: 141-147, 2014.

21 Choi AR, Jo MJ, Jung MJ, Kim HS and Yoon S: Selenate specifically sensitizes drug-resistant cancer cells by increasing apoptosis via $\mathrm{G}_{2}$ phase cell-cycle arrest without P-gp inhibition. Eur J Pharmacol 764: 63-69, 2015.
22 Choi AR, Kim JH, Cheon JH, Kim HS and Yoon S: Attenuation of colchicine toxicity in drug-resistant cancer cells by cotreatment with anti-malarial drugs. Anticancer Res 36: 58595866, 2016.

23 Choi AR, Kim JH, Woo YH, Kim HS and Yoon S: Anti-malarial drugs primaquine and chloroquine have different sensitization effects with anti-mitotic drugs in resistant cancer cells. Anticancer Res 36: 1641-1648, 2016.

24 Choi AR, Kim JH and Yoon S: Thioridazine specifically sensitizes drug-resistant cancer cells through highly increase in apoptosis and P-gp inhibition. Tumour Biol 35: 9831-9838, 2014.

$25 \mathrm{Kim} \mathrm{JH}$, Choi AR, Kim YK and Yoon S: Co-treatment with the anti-malarial drugs mefloquine and primaquine highly sensitizes drug-resistant cancer cells by increasing P-gp inhibition. Biochem Biophys Res Commun 441: 655-660, 2013.

26 Lim JS, Park Y, Lee BM, Kim HS and Yoon S: Co-treatment with Celecoxib or NS398 strongly sensitizes resistant cancer cells to antimitotic drugs independent of P-gp inhibition. Anticancer Res 36: 5063-5070, 2016.

27 Bhardwaj RK, Glaeser H, Becquemont L, Klotz U, Gupta SK and Fromm MF: Piperine, a major constituent of black pepper, inhibits human P-glycoprotein and CYP3A4. J Pharmacol Exp Ther 302: 645-650, 2002. 